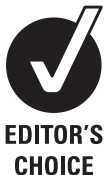

CHOICE

REVIEW

\title{
Update on the pathophysiology and management of idiopathic intracranial hypertension
}

\author{
Valérie Biousse, ${ }^{1,2}$ Beau B Bruce, ${ }^{1,2}$ Nancy J Newman ${ }^{1,2,3}$
}

${ }^{1}$ Department of Ophthalmology, Emory University, Atlanta, Georgia, USA

${ }^{2}$ Department of Neurology, Emory University, Atlanta, Georgia, USA

${ }^{3}$ Department of Neurological Surgery, Emory University, Atlanta, Georgia, USA

Correspondence to Dr V Biousse,

Neuro-Ophthalmology Unit, Emory Eye Center, 1365-B

Clifton Road NE, Atlanta, GA 30322, USA;

vbiouss@emory.edu

Received 13 December 2011 Revised 20 January 2012 Accepted 25 January 2012 Published Online First 15 March 2012

\section{ABSTRACT}

Idiopathic intracranial hypertension is a disease of unknown aetiology, typically affecting young obese women, producing a syndrome of increased intracranial pressure without identifiable cause. Despite a large number of hypotheses and publications over the past decade, the aetiology is still unknown. Vitamin A metabolism, adipose tissue as an actively secreting endocrine tissue and cerebral venous abnormalities are areas of active study regarding the pathophysiology of idiopathic intracranial hypertension. There continues to be no evidence based consensus or formal guidelines regarding management and treatment of the disease. Treatment studies show that the diagnostic lumbar puncture is a valuable intervention beyond its diagnostic importance, and that weight management is critical. However, many questions remain regarding the efficacy of acetazolamide, CSF shunting procedures and cerebral transverse venous sinus stenting.

Idiopathic intracranial hypertension (IIH) is a syndrome characterised by increased intracranial pressure (ICP) of unknown cause (box 1). By definition, the term 'IIH' describes patients with isolated raised ICP-that is, not related to an intracranial disorder, a meningeal process or cerebral venous thrombosis. ${ }^{2}$ However, patients who develop a syndrome of raised ICP triggered by certain medications or who are found to have cerebral transverse venous sinus stenoses (not thrombosis) are still conventionally classified as having 'IIH' (figure 1). Therefore, although imperfect, the term 'IIH' is currently the preferred designation for this disorder in the English literature, to the exclusion of 'pseudotumour cerebri' (often including patients with other causes of raised ICP such as cerebral venous thrombosis) and 'benign intracranial hypertension' (erroneously reassuring considering that a number of $\mathrm{IIH}$ patients irreversibly lose vision)..$^{2-7}$ This imperfect nomenclature will need to be revised as our understanding of the aetiology and pathophysiology of IIH improves. Although it has previously been emphasised that brain imaging should be normal in IIH patients, progress in non-invasive brain and vascular imaging has changed the radiological description of increased ICP, including IIH (box 2, figure 2). ${ }^{8-12}$

Although a relatively rare disease, IIH is becoming an important area of clinical research and activity, mostly because of the rising prevalence of obesity. ${ }^{4-6}$ Indeed, it was recently suggested that the economic costs of IIH in the USA exceed $\$ 444$ million dollars per year, mostly because of frequent hospital admissions, unsatisfactory treatment options and lost productivity of young patients. ${ }^{13}$ The rising incidence of obesity in the world will likely make the prevalence of IIH increase, resulting in further increased IIH related expenses.

There are multiple theories regarding the pathophysiology of this disorder but there is no unifying hypothesis. $^{3-6}$ There continues to be no evidence based consensus or formal guidelines regarding management and treatment of the disease although a clinical trial (discussed below) is currently ongoing in the USA (http://www.nordicclinicaltrials.com/). This review emphasises recent studies regarding the epidemiology, pathophysiology and management of IIH.

\section{EPIDEMIOLOGICAL DATA RELEVANT TO THE PATHOPHYSIOLOGY OF IIH}

IIH occurs most frequently among obese women of childbearing age. A recent multicentre case control study of newly diagnosed women with $\mathrm{IIH}$ compared with women with other neuro-ophthalmological disorders showed a dose relationship of higher body mass index (BMI) associated with a greater risk of $\mathrm{IIH}^{14}$ Interestingly, this study also showed that even non-obese patients (BMI <30) were at greater risk for IIH if they had a recent moderate weight gain (5-15\% of their weight). Another recent study also showed that a group of 26 women with recurrent IIH had a greater BMI at the time of recurrence compared with their BMI at the time of initial diagnosis. ${ }^{15}$ This study also demonstrated a greater degree of weight gain between initial resolution and recurrence compared with a group of $24 \mathrm{IIH}$ women without recurrence. Furthermore, patients without recurrence had stable weights, while patients with recurrence had a $6 \%$ weight gain, suggesting that even moderate weight gain might be a risk factor for recurrence. Additionally, in a more recent study, increasing degrees of obesity were associated with an increased risk of severe visual loss. ${ }^{16}$ A study from Israel suggested that gynaecoid (lower body) adiposity might be more associated with IIH than the more common visceral (abdominal or central) obesity, which is associated with cardiovascular diseases and diabetes. ${ }^{17}$ Although not all 40 obese women evaluated in this study had lower body adiposity, fat distribution in these IIH patients was clearly shifted to the lower body compared with age matched obese women living in the same country. The exact relationship between obesity and IIH remains poorly understood, with several 
Box 1 Modified Dandy criteria for the diagnosis of idiopathic intracranial hypertension

1. Signs and symptoms of increased intracranial pressure (headaches, nausea, vomiting, transient visual obscurations, papilloedema).

2. No localising focal neurological signs except unilateral or bilateral sixth nerve paresis.

3. CSF opening pressure $\geq 25 \mathrm{~cm}$ of water* but without cytological or chemical abnormalities.

4. Normal neuroimaging adequate to exclude cerebral venous thrombosis - that is, MRI of the brain, often with additional sequences (CT or MR venography).

*The number of $25 \mathrm{~cm}$ of water is not an absolute cut-off, especially in children in whom CSF opening pressures as high as $28 \mathrm{~cm}$ of water have been documented to be normal ${ }^{1}$

aetiological hypotheses proposed, including increased central venous pressure and various hormonal and metabolic changes commonly found in obese patients. ${ }^{18}$ Although there is no doubt that obesity plays a major role in the occurrence and, likely, the pathophysiology of IIH, many non-obese patients of either gender also develop a syndrome of isolated intracranial hyper-

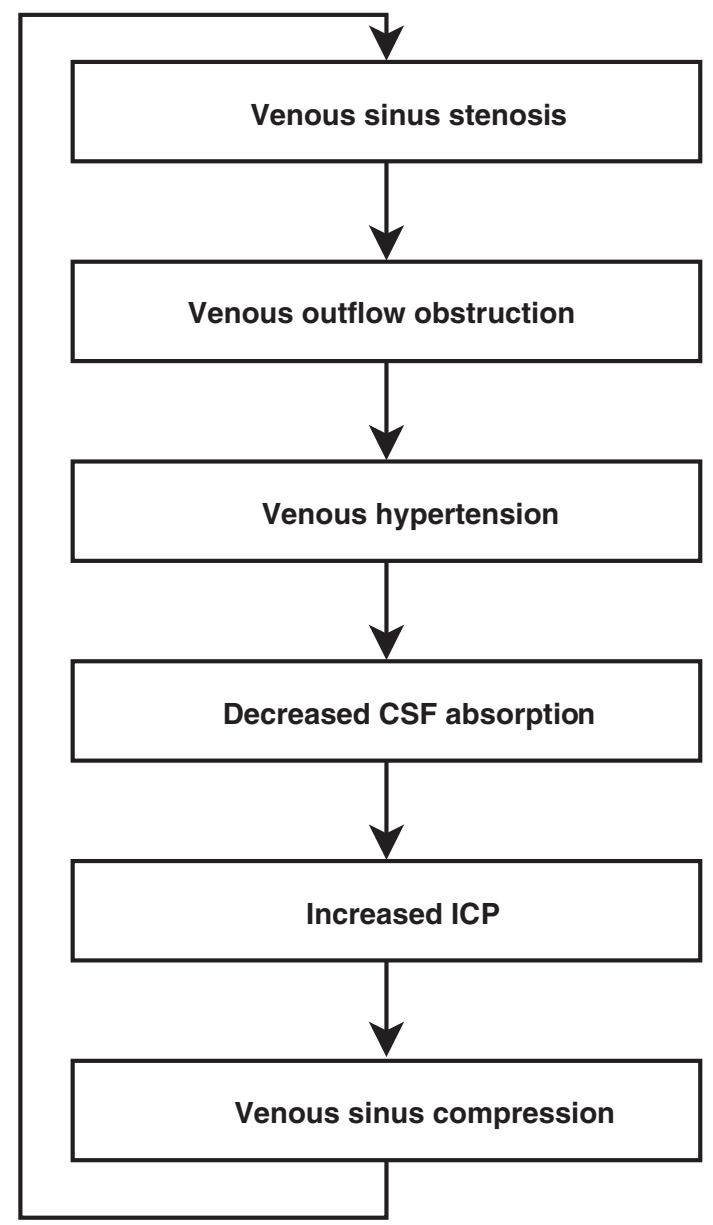

Figure 1 Mechanism by which transverse sinus stenosis leads to increase intracranial pressure. ICP, intracranial pressure.
Box 2 Brain and vascular imaging findings in patients with idiopathic intracranial hypertension

- Flattening of the posterior pole of the eyes

- Dilation and tortuosity of the optic nerve sheaths

- Empty sella turcica

- Stenosis of one or both transverse cerebral venous sinuses

Although common in idiopathic intracranial hypertension (IIH), these radiological findings are not specific to IIH and are also found in patients with other causes of raised intracranial pressure (ICP). Additionally, patients with normal ICP are occasionally incidentally found to have one or all of the above radiological findings.

tension similar to IIH. ${ }^{19}{ }^{20} \mathrm{IIH}$ is rare in prepubertal children and has characteristics distinct from the adult form, including no apparent predilection for obese girls. After puberty, however, the rate of obesity and the gender predilection is similar to that in the adult IIH population. ${ }^{1}$ A recent large series confirmed that about $10 \%$ of IIH patients are men. ${ }^{20}$ While affected men have a similar BMI compared with affected women, they are, on average, about a decade older than women at the time of presentation. ${ }^{20}$ Although race does not seem to influence the incidence of IIH, a recent study questioned whether the association between obesity and development of IIH among Asians is as strong as in other populations. ${ }^{21}$ Instead of affecting prevalence, race appears to be an important determining factor of a patient's visual prognosis, worse in Black patients compared with White patients living in the USA, ${ }^{22}$ and worse in White US IIH patients compared with white French IIH patients. ${ }^{23}$ Similarly, although men develop IIH less frequently than women, their visual prognosis is worse, perhaps because they have fewer headaches to alert them to the problem. ${ }^{20}$

Although various medications have been proposed to cause or, more likely, precipitate IIH (such as tetracycline and its derivatives, cyclosporine, lithium, nalidixic acid, nitrofurantoin, oral contraceptives, levonorgestrel, danaxol and tamoxifen), ${ }^{4}$ there are compelling test-retest data in few individual patients (such as in patients treated with tetracyclines, particularly minocycline). ${ }^{24-26}$

Obstructive sleep apnoea (OSA) is associated with $\mathrm{IIH}^{4}{ }^{4}$ It is unclear, however, if obesity is the common pathophysiological link or if OSA is another factor capable of triggering IIH in predisposed patients. It is important to emphasise that OSA might provide a worse prognosis for IIH patients. ${ }^{20} 27$ Routine screening of IIH patients with the Berlin questionnaire for OSA (brief and validated screening questionnaire which includes questions about snoring, daytime somnolence, BMI and hypertension) has been shown to be a reliable metric in the young population commonly affected by $\mathrm{IIH}^{28}$ Overnight polysomnography is usually obtained when the clinical suspicion for OSA is high in IIH patients. ${ }^{27} 28$

\section{PATHOPHYSIOLOGY OF IIH}

The pathophysiological mechanisms underlying the raised ICP in IIH remain unclear but those proposed classically include increased brain water content, excess CSF production, reduced CSF absorption and increased cerebral venous pressure. As emphasised, any pathophysiological theory must ultimately account for the remarkable predilection IIH has for obese young 
Figure $2 \mathrm{MRI}$ findings in idiopathic intracranial hypertension. Top: Posterior flattening of the globes resulting in shortening of the eyes and hyperopic shift (arrows). Dilation and tortuosity of the optic nerve sheath (arrowheads) Bottom left: Empty sella (arrow) Bottom right: Contrast-enhanced magnetic resonance venography showing bilateral distal transverse sinus stenoses (arrows).

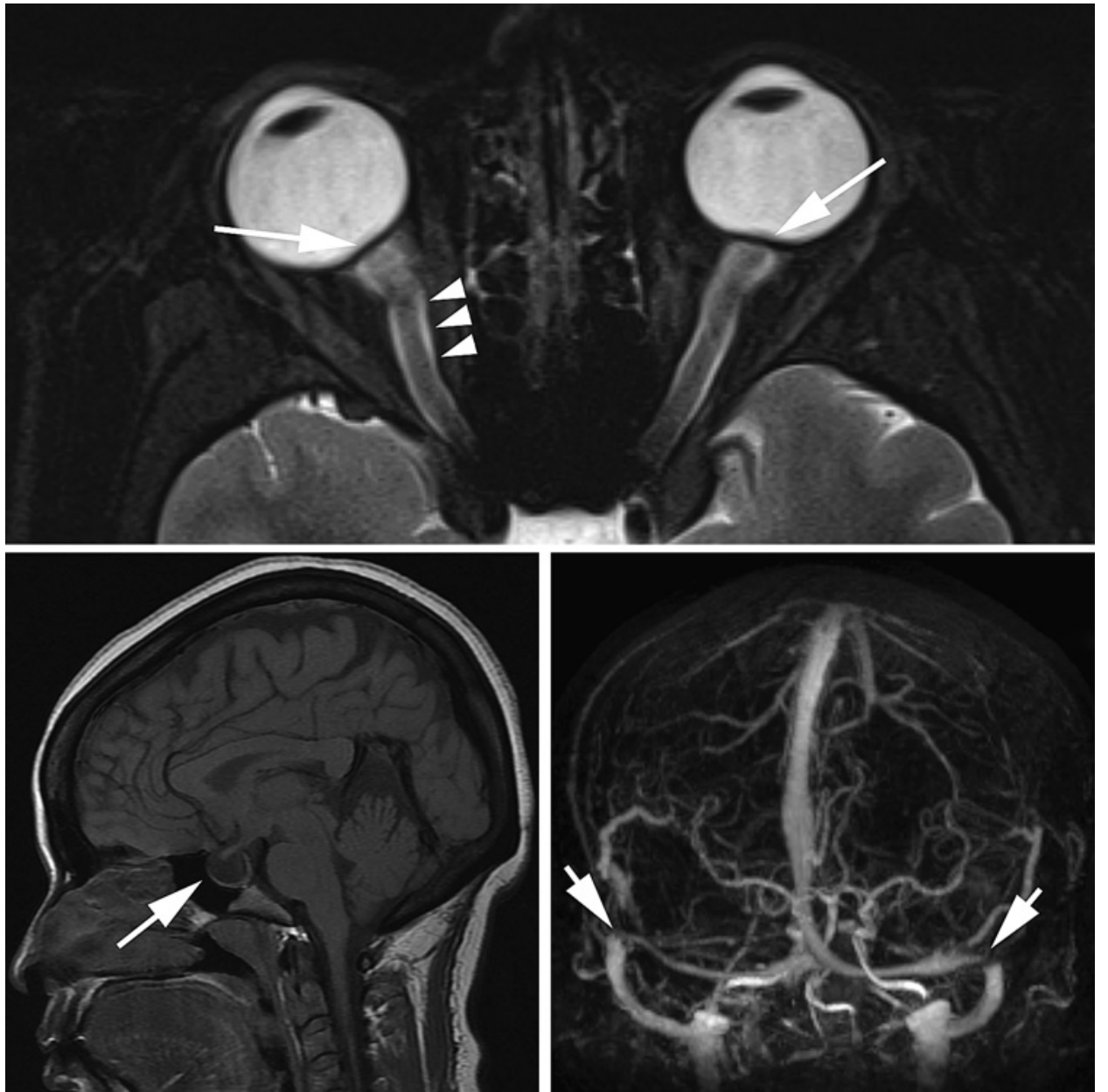

women, as well as the few epidemiological observations described above. Multiple coexisting mechanisms are likely necessary to produce the syndrome of isolated intracranial hypertension. It seems that some patients in particular (such as obese patients, especially young women, and those with anomalous distal transverse sinuses resulting in bilateral transverse sinus stenoses (TSS)) are predisposed to developing raised ICP which might be triggered by specific events or situations, such as weight gain, endocrine changes, hypercoagulable states, specific medications and OSA.

One longstanding hypothesis for the pathogenesis of IIH involves abnormal vitamin A metabolism. While early studies evaluating serum levels of vitamin A found conflicting evidence for the role of vitamin $\mathrm{A}^{29}$ two studies have shown elevated retinol levels in the CSF of patients with IIH. ${ }^{30} 31$ One of these studies also demonstrated that patients with IIH have higher levels of serum, but lower levels of CSF, retinol binding protein. ${ }^{31}$ However, despite a large number of publications in the field of IIH over the past few years, we could not find any major study regarding vitamin A metabolism published since 2007. The ongoing US IIH trial (http://www.nordicclinicaltrials.com/) will likely provide useful information on this topic since vitamin A measurements are obtained on included patients.

These observations regarding vitamin A may be linked to another area of emerging interest in endocrinology and $\mathrm{IIH},{ }^{18}$ the nature of adipose tissue as an actively secreting endocrine tissue. $^{32}$ In particular, adipose tissue derived retinol binding protein is released from adipose tissue and acts as a modulator of insulin sensitivity. ${ }^{29}$ Other adipose produced cytokines, such as leptin, have been implicated in the pathophysiology of IIH but their role remains unclear. ${ }^{18}$ Because the hormonal secretions and biological functions of adipose tissue are highly dependent on its regional distribution in the body, ${ }^{32}$ fat distribution may ultimately be as important as total adiposity in the pathogenesis of $\mathrm{IIH}^{17}$ However, the finding of predominant lower body adiposity in IIH is somewhat surprising given that IIH has been potentially associated with conditions related to increased intraabdominal visceral fat, such as elevated levels of adipose tissue derived retinol binding protein, ${ }^{30}$ polycystic ovarian disease in women ${ }^{33}$ and androgen deficiency in men. ${ }^{34}$ Another recent area of research includes the brain water channel aquaporin-4, which mediates rapid rapid transmembrane osmotic movement of water and is critically involved in brain water homeostasis. So far, results of studies investigating aquaporin-4 have been negative. ${ }^{35}{ }^{36}$ Metabolomics are also a promising area of study and aim to identify new candidate markers that may be involved in the pathophysiology of $\mathrm{IIH}^{37}{ }^{38} \mathrm{~A}^{\text {study }}{ }^{38}$ suggested that nuclear MR spectroscopic metabolic profiling of CSF and serum can identify differences between IIH, multiple sclerosis, cerebrovascular diseases and mixed neurological diseases. Identification of disease unique metabolites may also provide information on disease pathology and open new areas of research.

Increased intracranial venous pressure related to stenosis of the distal portion of the transverse cerebral sinuses is another proposed mechanism of IIH that has received substantial recent interest. $^{4-11}{ }^{39-42}$ Because CSF is passively resorbed into the intracranial venous sinuses via the arachnoid granulations, stenosis of a dominant transverse sinus or stenoses of both 
transverse sinuses can impair venous drainage, resulting in cerebral venous hypertension and impaired CSF absorption (figure 1). Numerous recent publications have shown that such TSSs are present in a large majority of patients with IIH. It is, however, not clear whether they are incidental, secondary to increased ICP or causal. ${ }^{40} 4344$ Anatomical studies have suggested that these stenoses might be incidentally related to the presence of trabeculae, septa or large arachnoid granulations in the transverse sinuses. ${ }^{39}$ It has also been shown that such distal TSS can develop secondary to increased ICP because of external compression of the distal portion of the transverse venous sinuses. The observation of complete reversal of TSS after a lumbar puncture or a CSF shunting procedure supports this theory (figure 3). ${ }^{43} 44$ In addition, TSS are occasionally found in patients without intracranial hypertension, suggesting that they may have no functional significance in some patients. ${ }^{8}$ Regardless of the cause of the TSS often observed in IIH patients, treatment with endovascular stenting usually results in decreased venous pressure gradient, thereby inducing improved CSF absorption and subsequent decrease in ICP (figure 3). ${ }^{43-45}$ It is still unclear, however, whether the presence of unilateral or bilateral TSS has any direct consequence on the natural history and prognosis of IIH.

Another venous mechanism proposed in the development of increased ICP in IIH patients is the occurrence of microthrombosis from thrombophilia within the cerebral veins, resulting in impaired CSF absorption. ${ }^{46-48}$ While many would argue that the presence of any thrombophilia would make these cases ineligible for the diagnosis of IIH, the absence of true venous sinus thrombosis and the strength of the associations between underlying systemic hypercoagulability disorders and so-called IIH in these studies is provocating. However, routine hypercoagulation studies in presumed IIH patients are not warranted unless there is a demonstrated clot within the cerebral venous sinuses or a strong personal or family history of thrombophilia. ${ }^{49}$

\section{TREATMENT OF IIH}

The two goals of treatment in IIH are to alleviate symptoms of increased ICP, particularly headaches, and to preserve vision.
General recommendations include evaluation and treatment of potential contributing factors (including weight gain and obesity, medication use, anaemia and OSA), weight loss and medical headache management. Immediate management is primarily based on the duration of symptoms, evaluation of visual function and patient characteristics (box 3).

The treatment of raised ICP itself begins with the diagnostic lumbar puncture, which is often effective in transiently improving symptoms and signs. Interestingly, it is not uncommon to observe a lasting clinical remission following a single lumbar puncture in some IIH patients, ${ }^{50}$ obviating the need for further medical or surgical treatment. This phenomenon cannot be simply explained by the amount of CSF drained, or by the hole made in the dura by the needle used for the lumbar puncture. Mathematical models of CSF hydraulics likely explain this phenomenon by the interaction of various factors, such as CSF formation, compliance, cerebral blood flow and outflow resistance, which can lead to multiple stable and unstable equilibrium levels of ICP in IIH. ${ }^{40}$ If a single lumbar puncture lowers the CSF pressure beyond the threshold level of an unstable equilibrium, the ICP must settle into a lower stable pressure state until other factors cause the pressure to exceed that threshold. This observation emphasises the importance of the initial lumbar puncture as a therapeutic procedure in $\mathrm{IIH}$ in addition to its diagnostic importance. It also explains why some patients dramatically improve after two or three lumbar punctures or require episodic lumbar punctures to remain asymptomatic.

Patients with persistent symptoms and signs can be treated using medical and surgical approaches, although there are currently no results from large randomised controlled trials prospectively assessing and comparing these treatments.

Weight loss is a critical part of the treatment of overweight and obese IIH patients. Only a modest degree of weight loss (about $5-10 \%$ of total body weight) is usually required for improvement in symptoms and signs ${ }^{51}$ although it is likely that aggressive weight control is an efficient way to improve the overall quality of life of obese IIH patients. A recent study of 25 obese women with IIH showed that weight loss effectively reduced not only headaches and papilloedema, but also ICP. ${ }^{52}$
Figure 3 Resolution of bilateral transverse sinus stenosis after lumboperitoneal shunt in a young obese woman with idiopathic intracranial hypertension.

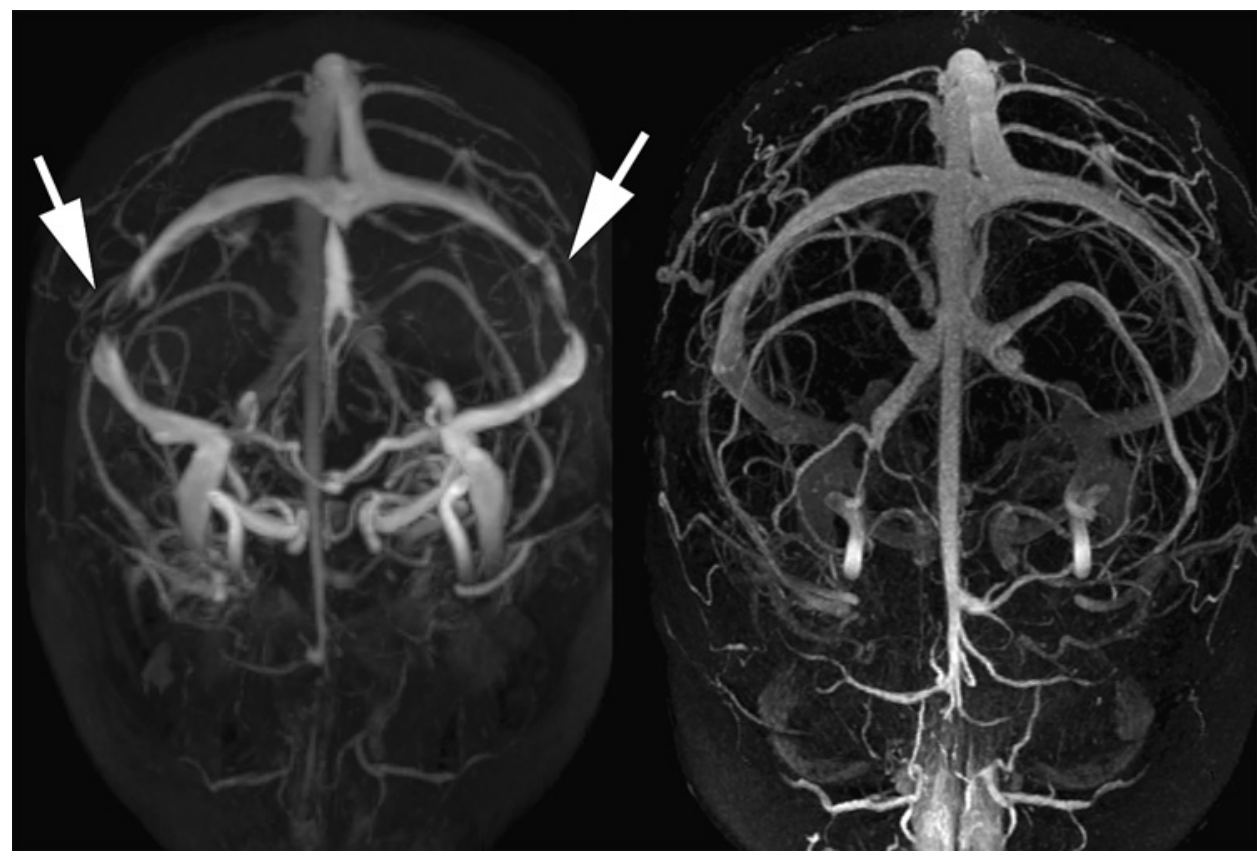


Box 3 Factors independently associated with a worse visual outcome in idliopathic intracranial hypertension

Male gender

- Race (Black patients)

- Morbid obesity

- Anaemia

- Obstructive sleep apnoea

- Acute onset of symptoms and signs of raised intracranial pressure (fulminant idiopathic intracranial hypertension)

Weight loss is often not an effective short term treatment and thus usually must be initiated in association with other treatments, However, it remains essential to emphasise the importance of weight loss and the prevention of weight fluctuation in the long term management of obese or overweight IIH patients in order to minimise the risk of recurrence. ${ }^{15}$ Bariatric surgery can be considered in morbidly obese IIH patients in whom attempts at weight loss have been unsuccessful or in whom other medical morbidities of obesity already coexist, and two recent studies have specifically reviewed the positive effects of bariatric surgery on IIH. $^{53} 54$

Carbonic anhydrase inhibitors, such as acetazolamide (1-2 g daily, most often divided into two doses), are the main medical treatment classically prescribed for IIH. Acetazolamide decreases the production of CSF in humans and therefore has always been considered the treatment of choice in $\mathrm{IIH}^{55}$ although no trial data are currently available to confirm its effectiveness. The preliminary results of the IIH Pilot Trial from Birmingham, UK, in which 50 patients were randomised to receive acetazolamide or no acetazolamide, emphasised the practical difficulties of performing such a study because of poor recruitment and medication side effects (paraesthesias, altered taste sensation and lethargy). ${ }^{56}$ A multicentre, double blind, placebo controlled clinical trial, called the IIHTT (the Idiopathic Intracranial Hypertension Treatment Trial), is currently enrolling patients in the USA (http://www.nordicclinicaltrials.com/). This trial compares the efficacy of acetazolamide and placebo in the treatment of IIH patients with moderate visual field defects. All patients are also treated with a low sodium diet and participate in a standardised weight loss programme. This trial will hopefully clarify the efficacy of acetazolamide and weight loss in IIH patients with moderate visual loss.

Topiramate (which has weak carbonic anhydrase inhibition properties) has also been suggested for the treatment of IIH, particularly for the treatment of headaches. ${ }^{57}$ A small open label study comparing acetazolamide with topiramate for the treatment of IIH suggested that topiramate might be effective in the treatment of IIH. Presumed mechanisms of action of topiramate in IIH were a combination of weight reduction as well as reduction of CSF formation. ${ }^{57}$

A small study ${ }^{58}$ suggested that octreotide, a potent inhibitor of growth hormone and insulin-like growth factor 1, might reduce the ICP in IIH patient. Although this drug is not routinely used in IIH, it might represent a promising area of research.

Oral steroids have been used as a treatment for IIH in the past but are associated with significant long term side effects, such as weight gain, obviously undesirable in this patient population, and therefore should not be prescribed. High dose intravenous steroids are still occasionally used in patients with rapidly progressive visual loss from fulminant IIH while a more definitive treatment is organised. ${ }^{59}$

Surgery is required in patients with a fulminant onset of disease or when other treatments have failed to prevent progressive visual loss. More rarely, surgery may be performed for refractory headaches related to chronically elevated ICP. The choice of procedure depends on local resources, as well as the patient's symptoms and signs. In patients with papilloedema who have severe visual loss, but minimal or no headache, optic nerve sheath fenestration (ONSF) is often advised, while in those with visual loss, papilloedema and headache, a CSF diversion procedure, such as ventriculo-peritoneal (VP) or lumbo-peritoneal (LP) shunting, is preferred. Aggressive management with ONSF or CSF shunting is usually required to prevent catastrophic visual loss in those with acute and rapidly progressive visual loss. ${ }^{59}$ These patients might benefit from a transient lumbar drain while awaiting a more definite surgical procedure. When available, an ONSF is often performed first in patients with visual loss and papilloedema. Although no prospective study has effectively compared LP shunts with VP shunts, a large number of recent publications have addressed surgical interventions of $\mathrm{IIH}^{60-66}$ Retrospective series suggest that both techniques are equally efficient in controlling clinical manifestations of IIH as well as reducing the intracranial pressure in the immediate postoperative period. In one series, ${ }^{65}$ failure rates seemed to be slightly higher for VP shunts (14\%) than LP shunts (11\%). However, revision rates were higher with LP shunts (60\%) than with VP shunts (30\%). Despite the apparent high rate of complications (and failure), CSF shunting procedures remain the most widely performed surgical treatment for IIH and are usually very useful acutely to prevent or treat devastating visual loss in selected patients. It is important to emphasise that most IIH patients have a relatively 'benign' and self-limited course, and never require any surgical treatment.

It is now well accepted that stenting of transverse venous sinus stenoses reduces cerebral venous pressure, reduces ICP and improves symptoms and signs in selected IIH patients. ${ }^{43} 4567$ However, endovascular venous sinus stenting can result in serious complications, such as stent migration, venous sinus perforation, in-stent thrombosis, subdural haemorrhage and the development of recurrent stenoses immediately proximal to the stent. ${ }^{43} 4567$ Until more data are available on the safety and long term outcome of venous stenting in young IIH patients, this procedure should be limited to selected patients with bilateral TSS or with a hypoplastic transverse sinus on one side and TSS on the other, and refractory symptoms and signs of increased ICP, who cannot undergo (or have failed) more conventional surgical treatments.

\section{CONCLUSION}

Many questions remain unanswered about IIH. Its association with female gender and obesity is striking. However, studies indicate that IIH can also occur in men, non-obese adults, older adults and in prepubertal children. Identification of subgroups at high risk for irreversible visual loss, such as Black patients, men and patients with fulminant IIH (box 3), helps determine management approaches and refine follow-up strategies. Ongoing pathophysiology studies, as well as clinical trials, are promising and should provide more insight into this relatively common, yet poorly understood, syndrome of isolated intracranial hypertension. 
Contributors All three authors contributed to the review of the literature, and the preparation of the manuscript and figures.

Funding This study was supported in part by a departmental grant (Department of Ophthalmology) from Research to Prevent Blindness Inc, New York, by core grant P30-EY06360 (Department of Ophthalmology). BBB receives research support from the NIH/PHS (KL2-RR025009, UL1-RR025008), NIH/NEI (K23-EY019341) and the Knights Templar Eye Foundation; and received the American Academy of Neurology Practice Research Fellowship. NJN is a recipient of the Research to Prevent Blindness Lew R Wasserman Merit Award.

Competing interests None

Provenance and peer review Commissioned; externally peer reviewed.

\section{REFERENCES}

1. Ko MW, Liu GT. Pediatric idiopathic intracranial hypertension (pseudotumor cerebri). Horm Res Paediatr 2010;74:381-9.

2. Friedman DI, Jacobson DM. Diagnostic criteria for idiopathic intracrania hypertension. Neurology 2002;59:1492-5.

3. Friedman DI, Jacobson DM. Idiopathic intracranial hypertension. J Neuroophthalmol 2004:24:138-45.

4. Wall M. Idiopathic intracranial hypertension. Neurol Clin 2010;28:593-617.

5. Digre KB. Idiopathic intracranial hypertension. BMJ 2010;341:c2836.

6. Kapoor KG. More than meets the eye? Redefining idiopathic intracranial hypertension. Int J Neurosci 2010;120:471-82.

7. Karahalios DG, Rekate HL, Khayata MH, et al. Elevated intracranial venous pressure as a universal mechanism in pseudotumor cerebri of varying etiologies. Neurology 1996; 46:198-202

8. Farb RI, Vanek I, Scott JN, et al. Idiopathic intracranial hypertension: the prevalence and morphology of sinovenous stenosis. Neurology 2003;60:1418-24.

9. Degnan AJ, Levy LM. Pseudotumor cerebri: brief review of clinical syndrome and imaging findings. AJNR Am J Neuroradiol 2011;32:1986-93.

10. Rohr AC, Riedel C, Fruehauf MC, et al. MR imaging findings in patients with secondary intracranial hypertension. AJNR Am J Neuroradiol 2011:32:1021-9.

11. Connor SE, Siddiqui MA, Stewart VR, et al. The relationship of transverse sinus stenosis to bony groove dimensions provides an insight into the aetiology of idiopathic intracranial hypertension. Neuroradiology 2008;50:999-1004.

12. Shofty B, Ben-Sira L, Constantini S, et al. Optic nerve sheath diameter on MR imaging: Establishment of norms and comparison of pediatric patients with idiopathic intracranial hypertension with healthy controls. AJNR Am J Neuroradiol. 2012:33:366-9

13. Friesner D, Rosenman R, Lobb BM, et al. Idiopathic intracranial hypertension in the USA: the role of obesity in establishing prevalence and healthcare costs. Obes Rev 2011:12:e372-80

14. Daniels AB, Liu GT, Volpe NJ, et al. Profiles of obesity, weight gain, and quality of life in idiopathic intracranial hypertension (pseudotumor cerebri). Am J Ophthalmol 2007:143:635-41.

15. Ko MW, Chang SC, Ridha MA, et al. Weight gain and recurrence in idiopathic intracranial hypertension: a case-control study. Neurology 2011;76:1564-7.

16. Szewka AJ, Bruce BB, Newman NJ, et al. Idiopathic intracranial hypertension: relation between obesity and visual outcomes. J Neuroophthalmol. Published Online First: 3 January 2012

17. Kesler A, Kliper E, Shenkerman G, et al. Idiopathic intracranial hypertension is associated with lower body adiposity. Ophthalmology 2010;117:169-74.

18. Ooi LY, Walker BR, Bodkin PA, et al. Idiopathic intracranial hypertension: can studies of obesity provide the key to understanding pathogenesis? Br J Neurosurg. 2008; 22:187-94

19. Bruce BB, Kedar S, Van Stavern GP, et al. Atypical idiopathic intracranial hypertension: normal BMI and older patients. Neurology 2010;74:1827-32.

20. Bruce BB, Kedar S, Van Stavern GP, et al. Idiopathic intracranial hypertension in men. Neurology 2009;72:304-9.

21. Kim TW, Choung HK, Khwarg Sl, et al. Obesity may not be a risk factor for idiopathic intracranial hypertension in Asians. Eur J Neurol 2008;15:876-9.

22. Bruce BB, Preechawat P, Newman NJ, et al. Racial differences in idiopathic intracranial hypertension. Neurology 2008; 70:861-7.

23. Mrejen S, Vignal C, Bruce BB, et al. Idiopathic intracranial hypertension: a comparison between French and North-American white patients. Rev Neurol (Paris) 2009;165:542-8.

24. Friedman DI. Medication-induced intracranial hypertension in dermatology. $A m ~ J$ Clin Dermatol 2005;6:29-37.

25. Kesler A, Goldhammer Y, Hadayer A, et al. The outcome of pseudotumor cerebri induced by tetracycline therapy. Acta Neurol Scand 2004:110:408-11.

26. Chiu AM, Chuenkongkaew WL, Cornblath WT, et al. Minocyclin treatment and pseudotumor cerebri syndrome. Am J Ophthalmol 1998;126:116-21.

27. Wall M, Purvin V. Idiopathic intracranial hypertension in men and the relationship to sleep apnea. Neurology 2009;72:300-1.

28. Thurtell MJ, Bruce BB, Rye DB, et al. The Berlin questionnaire screens for obstructive sleep apnea in idiopathic intracranial hypertension. J Neuroophthalmol 2011;31:316-19.
29. Libien J, Blaner WS. Retinol and retinol-binding protein in cerebrospinal fluid: can vitamin A take the "idiopathic" out of idiopathic intracranial hypertension? J Neuroophthalmol 2007:27:253-7.

30. Tabassi A, Salmasi AH, Jalali M. Serum and CSF vitamin A concentrations in idiopathic intracranial hypertension. Neurology 2005;64:1893-6.

31. Warner JE, Larson AJ, Bhosale P, et al. Retinol-binding protein and retinol analysis in cerebrospinal fluid and serum of patients with and without idiopathic intracrania hypertension. J Neuroophthalmol 2007;27:258-62.

32. Wozniak SE, Gee LL, Wachtel MS, et al. Adipose tissue: the new endocrine organ? Dig Dis Sci 2009;54:1847-56.

33. Glueck CJ, Aregawi D, Goldenberg N, et al. Idiopathic intracranial hypertension, polycystic-ovary syndrome, and thrombophilia. J Lab Clin Med 2005; 145:72-82

34. Fraser JA, Bruce BB, Rucker J, et al. Risk factors for idiopathic intracranial hypertension in men: a case-control study. J Neurol Sci 2010:290:86-9.

35. Dhungana S, Waters P, Ismail A, et al. Absence of aquaporin-4 antibodies in patients with idiopathic intracranial hypertension. J Neurol 2010:257:1211-12. Corrected and republished in: J Neurol 2010:257:1229-30.

36. Kerty E, Heuser K, Indahl UG, et al. Is the brain water channel aquaporin-4 a pathogenetic factor in idiopathic intracranial hypertension? Results from a combined clinical and genetic study in a Norwegian cohort. Acta Ophthalmol Published Online First: 13 September 2011. doi:10.1111/j.1755-3768.2011.02231.x

37. Brettschneider J, Hartmann N, Lehmensiek V, et al. Cerebrospinal fluid markers of idiopathic intracranial hypertension: is the renin-angiotensinogen system involved? Cephalalgia 2011;31:116-21.

38. Sinclair AJ, Viant MR, Ball AK, et al. NMR-based metabolomic analysis of cerebrospinal fluid and serum in neurological disease - a diagnostic tool? NMR Biomed 2010:23:123-32.

39. Strydom MA, Briers N, Bosman MC, et al. The anatomical basis of venographic filling defects of the transverse sinus. Clin Anat 2010;23:153-9.

40. Bateman GA, Stevens SA, Stimpson J. A mathematical model of idiopathic intracranial hypertension incorporating increased arterial inflow and variable venous outflow collapsibility. J Neurosurg 2009:110:446-56.

41. Sander K, Poppert H, Etgen T, et al. Dynamics of intracranial venous flow patterns in patients with idiopathic intracranial hypertension. Eur Neurol 2011;66:334-8.

42. Rohr A, Bindeballe J, Riedel C, et al. The entire dural sinus tree is compressed in patients with idiopathic intracranial hypertension: a longitudinal, volumetric magnetic resonance imaging study. Neuroradiology 2012;54:25-33.

43. Ahmed R, Friedman Dl, Halmagyi GM. Stenting of the transverse sinuses in idiopathic intracranial hypertension. J Neuroophthalmol 2011;31:374-80.

44. Stienen A, Weinzierl M, Ludolph A, et al. Obstruction of cerebral venous sinus secondary to idiopathic intracranial hypertension. Eur J Neurol 2008;15:1416-18.

45. Albuquerque FC, Dashti SR, Hu YC, et al. Intracranial venous sinus stenting for benign intracranial hypertension: clinical indications, technique, and preliminary results. World Neurosurg 2011;75:648-52.

46. Kesler A, Kliper E, Assayag EB, et al. Thrombophilic factors in idiopathic intracrania hypertension: a report of 51 patients and a meta-analysis. Blood Coagul Fibrinolysis 2010;21:328-33

47. Hannerz J, Antovic JP, Blombäck M, et al. Inflammatory and haemostatic markers in idiopathic intracranial hypertension. J Intern Med 2011;270:496-9.

48. Biousse V, Rucker JC, Vignal C, et al. Anemia and papilledema. Am J Ophthalmol 2003:135:437-46.

49. Biousse V, Ameri A, Bousser MG. Isolated intracranial hypertension as the only sign of cerebral venous thrombosis. Neurology 1999;53:1537-42.

50. De Simone R, Marano E, Fiorillo C, et al. Sudden re-opening of collapsed transverse sinuses and longstanding clinical remission after a single lumbar puncture in a case of idiopathic intracranial hypertension. Pathogenetic implications. Neurol Sci 2005;25:342-4.

51. Wong R, Madill SA, Pandey P, et al. Idiopathic intracranial hypertension: the association between weight loss and the requirement for systemic treatment. BMC Ophthalmol 2007;7:15

52. Sinclair AJ, Burdon MA, Nightingale PG, et al. Low energy diet and intracranial pressure in women with idiopathic intracranial hypertension: prospective cohort study. BMJ 2010;341:c2701.

53. Egan RJ, Meredith HE, Coulston JE, et al. The effects of laparoscopic adjustable gastric banding on idiopathic intracranial hypertension. Obes Surg 2011;21:161-6.

54. Fridley J, Foroozan R, Sherman V, et al. Bariatric surgery for the treatment of idiopathic intracranial hypertension. J Neurosurg 2011;114:34-9.

55. Rubin RC, Henderson ES, Ommaya AK, et al. The production of cerebrospinal fluid in man and its modification by acetazolamide. J Neurosurg 1966:25:430-6.

56. Ball AK, Howman A, Wheatley $\mathrm{K}$, et al. A randomised controlled trial of treatment for idiopathic intracranial hypertension. J Neurol 2011:258:874-81.

57. Celebisoy N, Gokcay F, Sirin H, et al. Treatment of idiopathic intracrania hypertension: topiramate vs acetazolamide, an open-label study. Acta Neurol Scand 2007:116:322-7.

58. Deftereos SN, Panagopoulos G, Georgonikou D, et al. Treatment of idiopathic intracranial hypertension: is there a place for octreotide? Cephalalgia 2011:31:1679-80.

59. Thambisetty M, Lavin PJ, Newman NJ, et al. Fulminant idiopathic intracranial hypertension. Neurology 2007;68:229-32. 
60. Sinclair AJ, Kuruvath S, Sen D, et al. Is cerebrospinal fluid shunting in idiopathic intracranial hypertension worthwhile? A 10-year review. Cephalalgia 2011;31:1627-33

61. Ulivieri S, Oliveri G, Georgantzinou M, et al. Long-term effectiveness of lumboperitoneal flow-regulated shunt system for idiopathic intracranial hypertension. J Neurosurg Sci 2009;53:107-11.

62. El-Saadany WF, Farhoud A, Zidan I. Lumboperitoneal shunt for idiopathic intracranial hypertension: patients' selection and outcome. Neurosurg Rev 2012:35:239-44.

63. Kandasamy J, Hayhurst C, Clark S, et al. Electromagnetic stereotactic ventriculoperitoneal CSF shunting for idiopathic intracranial hypertension: a successful step forward? World Neurosurg 2011;75:155-60.
64. Tarnaris A, Toma AK, Watkins LD, et al. Is there a difference in outcomes of patients with idiopathic intracranial hypertension with the choice of cerebrospinal fluid diversion site: a single centre experience. Clin Neurol Neurosurg 2011;113:477-9.

65. Abubaker K, Ali Z, Raza K, et al. Idiopathic intracranial hypertension: lumboperitoneal shunts versus ventriculoperitoneal shunts-case series and literature review. Br J Neurosurg 2011:25:94-9.

66. Alsuhaibani $\mathbf{A H}$, Carter $\mathrm{KD}$, Nerad JA, et al. Effect of optic nerve sheath fenestration on papilledema of the operated and the contralateral nonoperated eyes in idiopathic intracranial hypertension. Ophthalmology 2011:118:412-14.

67. Ahmed RM, Wilkinson M, Parker GD, et al. Transverse sinus stenting for idiopathic intracranial hypertension: a review of 52 patients and of model predictions. AJNR Am J Neuroradiol 2011:32:1408-14.
Advancing Postgraduates. Enhancing Healthcare.

The Postgraduate Medical Journal is dedicated to advancing the understanding of postgraduate medical education and training.

- Acquire the necessary skills to deliver the highest possible standards of patient care

- Develop suitable training programmes for your trainees

- Maintain high standards after training ends

Published on behalf of the fellowship for Postgraduate Medicine

FOR MORE DETAILS OR TO SUBSCRIBE,

VISIT THE WEBSITE TODAY

postgradmedj.com 